\title{
Social Media Data Analysis using Recommendation Algorithms
}

\author{
S. Sathyavathi, K.R.Baskaran, S.Kavitha
}

\begin{abstract}
Recommender frameworks (RSs) are utilized in application areas to help clients in the quest for their preferred items . Recommender system filters information which takes users ratings and predict user preferences in ecommerce and other categorical websites. We examine individual proposal dependent on client inclinations and search the neighbors through the client inclinations. It generates recommendations based on implicit feedback or explicit feedback. Implicit feedback is based on analysis of browsing patterns of the user. Express criticism is produced from the appraisals given by the client. All the more extensively tended to was the subject of AI's calculations, centered around separating calculations dependent on the clients or questions, and dependent on substance.
\end{abstract}

Keywords - Recommendation System, text mining, decision making, Content-based Filtering (CBF).

\section{INTRODUCTION}

- Recommendation engines is a kind of work from a selected form of data filtering system technique that makes an attempt to gift data things (films, television, video on demand, music, books, news, images, web pages, etc) that are doubtless to be of interest to the user

- Typically, a recommender system compares the user's profile to some reference characteristics, Associate in Nursing the seeks to predict the 'rating' that a user would provide to an item that they had not nonetheless thoughtabout.

Recommendation Systems are the package of solutions available in online shopping sites to supply additional services to the customers purchasing through web by serving them to find the merchandise that they are interested. This will help the customer to remember about their purchase.The recommendation engine provide suggestion like recommending things to users with the supported samples of their preferences. Various on-line stores like Amazon, Snap deal, Flipkart give recommendations to customers for their purchase which in turn considerably increase their online sales. The basic approaches for recommending are :

- Collaborative Filtering (social filtering)

- Content-based Filtering

- Text Mining

As world wide web is the one of the powerful force of generating data in exponential manner .For the clients of those sites it turns out to be dynamically extreme and time

Revised Manuscript Received on September 14, 2019.

S. Sathyavathi, Assistant professor, Department of Information Technology, Kumaraguru College Of Technology, Coimbatore, Tamilnadu, India.

Dr.K.R.Baskaran, Professor ,Department of Computer Science and Engineering, Kumaraguru College Of Technology, Coimbatore, Tamilnadu, India.

S.Kavitha, Assistant professor, Department of Information Technology, Kumaraguru College Of Technology, Coimbatore, Tamilnadu, India. exceptional to search out the information they're scanning for. to help clients understand the learning that is as per their inclinations a web site will be customized. Recommender structures will improve a site for individual customers by intensely including hyperlinks. The CBF approach recommends merchandise to focus on customers consistent with the likeliness of their neighbors. Content-based filtering analyzes the content of knowledge sources (e.g., the markup language supply of net pages) that are rated to form a profile of the user's interests in terms of regularities within the content of the knowledge that was rated extremely. it's inhibitive to estimate the preference similarities between variety of customers. As an example, similar preferences might be outlined as the preferences of shoppers UN agency have similar ratings of things. it's tough to get the correct client ratings of merchandise particularly once special information is required for rating.

The Second approach is cooperative filtering approach. cooperative approaches realize and suggest data sources for a private user that are rated extremely by different users UN agency have a pattern of ratings like that of the user. This approach suggest merchandise to customers by examining the shopping pattern of different customers. It recommends the product for purchase to the customer based on the pattern of customer related to a customer's shopping pattern.

The third approach could be a book mining- based suggestion framework that helps clients in their higher subjective procedure in on-line item customization. The content mining-based Recommendation System doesn't deliver neighborhoods in its suggestion technique, and henceforth, its proposal procedure is independent of past customer profiles. It's therefore ready to produce right suggestions even once explicit customer evaluations are disseminated, in this manner mitigating the deficiency issue of standard agreeable sifting based frameworks.

The content of paper is organized to summarize the recommendation techniques like Content based, Collaborative based and text based approaches.

\section{CONTENT-BASED FILTERING (CBF)}

This filtering technique is that the most well liked approach for building recommendation systems. CF-based systems suggest merchandise to purchase or shop a product by using the credit rating (Score) for the product provided by the merchandise for the product they are likely to purchase. The system creates a group of neighbors i.e. list of customers who have previously purchased the same items 
which the customer looks for purchase. The target users will be served with the suggestions provided by the customers (ie product rating) for the product which the customer looks for purchase at present. The similar group of people with same interest of purchase pattern will be developed to provide suggestion to the user for better and efficient purchase according to their needs.

Every massive assortment wants a particular structure to create it straightforward for guests to seek out what they're searching for. A web site will be structured by dividing its web content into two pages like content pages and navigation pages. The content pages provides the user with the information about their interested things for purchase .The navigation pages facilitate the user to look for the interested things for their purchase. The division cannot be a strict classification.. Moreover, a navigation page for one user might be a content page to other user and vice versa. Generally this division provides the way of describing the structure of a web site for a particular product .It also provides the method for improving individual users interest dynamically adding hyperlinks to connect to the neighboring customers.

Thus the below structure illustrates the content based filtering approach pictorially. The below table provides the information about each picture in the below structure.

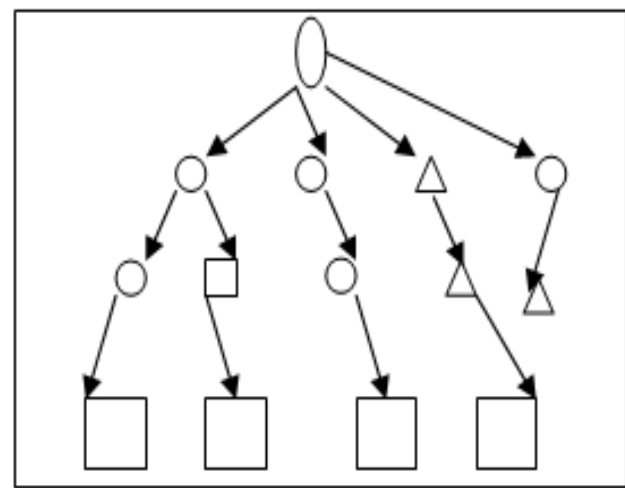

\begin{tabular}{|l|l|}
\hline Navigation Page & \\
\hline Content Page & \\
\hline Hybrid Page & \\
\hline Hypertext Link & \\
\hline
\end{tabular}

A substance based sifting framework chooses things bolstered the relationship be tween's the substance of the things |the things $\}$ and consequently the client's inclinations as basic an agreeable separating framework that picks things upheld the connection be tween's people with comparative inclinations. It makes proposals by examination a client profile with the substance of each archive inside the variety. The substance of an archive will be painted with a gathering of terms. Terms are removed from records by going through assortment of parsing steps. first all markup language labels and stop (words that happen on a regular basis and can't be utilized as discriminators) are expelled. The rest of the words are decreased to their stem by expelling prefixes and postfixes [Porter 1980]. for instance the words "PC", "PCs" and "processing" may all be decreased to "comput".
Content-based sifting at the same time, contrasts from information recovery inside the way during which the interests of a client are painted. instead of utilizing an inquiry Associate in nursing information sifting framework attempts to show the client's for quite some time run interests

Basic idea: Documents with supported content and properties.

Profile: It consists of keywords with associated weights, for documents matching with the profile of the customer.

Recommendations: It provides recommendation to the supported document with information retrieval and knowledge filtering systems based on objective measurable properties and supported objective measurable properties.

Data recovery and data sifting frameworks play out all estimations on the single connection descriptor object. As Associate in nursing model, objects is content archives and descriptors is watchwords.

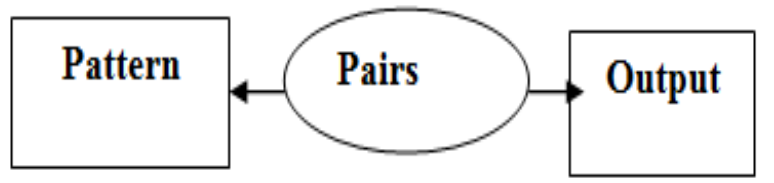

Fig 1: Content Based Information Retrieval

The basic operation of recommender systems is to obtain or filter object data. The Table 1 and Table 2 provides an example data to picturize the operations of filtering techniques.

The collection of object references for the data object is a tough job. Keyword based retrieval method is used for object -based query operations. Users' needs to provide input to the system as an keyword based format. The system in turn returns the output as +ve or -ve examples from the set of objects (relevance feedback). To consolidate the two info information type's watchwords and archives in a solitary question, reports are supplanted with the most expressive catchphrases utilized in these records. It is hard to acquire the precise client appraisals of items particularly when uncommon learning is required for rating.

\begin{tabular}{|l|l|l|}
\hline $\begin{array}{l}\text { Input data as } \\
\text { Keywords }\end{array}$ & $\begin{array}{l}\text { Data } \\
\text { Dictionary }\end{array}$ & Filtered Output \\
\hline Object & Indexing & $\begin{array}{l}\text { Query by } \\
\text { example }\end{array}$ \\
\hline & $\begin{array}{l}\text { Input data as } \\
\text { Keywords }\end{array}$ & Object \\
& & \\
\cline { 2 - 3 }
\end{tabular}

Table 1: content-based systems. Rows represents the input and column represents the output

\section{COLLABORATIVE PROCESSING (CF)}

The collaborative system the operations are based on single data relation with one or more entities. Figure 2 shows the relationship between entities. 


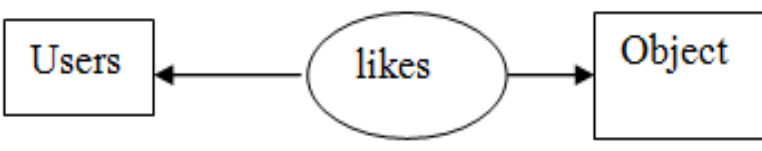

Fig 2: ER Model of Collaborative system

\begin{tabular}{|l|l|l|}
\hline Customer & Pair maker & $\begin{array}{l}\text { Collaborative filtering } \\
\text { in active mode }\end{array}$ \\
\hline Query & Find experts & $\begin{array}{l}\text { Collaborative filtering } \\
\text { in automated mode }\end{array}$ \\
\hline & Customer & Query \\
\cline { 2 - 3 }
\end{tabular}

Table 2: collaborative -based systems Rows represents the input and column represents the output

In passive collaborative filtering users are requested to fill their profile details with the ratings to the product or suggestions, and then the rating alone is used to query the system. In active collaborative system the customers are addressed separately for either providing them the recommendation or to gather the recommendations from them. A typical example is match finding /pair finding.(matrimony)

Community oriented sifting frameworks more often than not make two strides:

- Look for clients who offer a similar rating designs with the dynamic user(the client whom the forecast is for).

- Use the evaluations from those like - disapproved of clients found in step 1 to compute an expectation for the dynamic client

Shared separating methodologies prescribe items to clients by inspecting the acquisition of other similarly invested clients. However experiences two significant disadvantages, known as the sparsity and versatility issues [3]. The sparsity issue, because of inadequate past client profiles, prompts wrong item proposals. The versatility issue emerges since the proposal method of cooperative sifting based Recommendation Systems requires greater likeness counts when the quantity of past client profiles increments, in this way influencing the exhibition. Another confinement of collective sifting frameworks is that they may not precisely become familiar with the clients' inclinations. The majority of the methodologies proposed to manage these issues are either too computationally costly or incorrect. Some of the challenges in collaborative filtering are Cold Start, Sparsity, First Rater, and Popularity Bias. These challenges can be overcome using another approach text mining.

\section{TEXT MINING \& RESULTS}

This segment portrays the content mining (TM) based suggestion strategy. The TM-based recommendation system plays out its suggestion capacities by following a technique comprising of 5 phases - Collecting data, Data preparation, Similarity finding, Generation of recommendation and Final-Processing. Information is collected as document

Input collected is preprocessed for getting the knowledge. The system learns about the product from the documentations made available at the preprocessing stages
.The system provides suggestion and recommends the product to the users by pairing the information of purchase with available information of purchase of other users in the repository. Then the post processing stage is carried out to improve the accuracy for the providing recommendations to the customers.

\section{Collecting Data}

As the first step information is collected by the system about the items provided from the users purchase pattern to learn the product features. It performs the task by collecting information from online pages and then process the textual information into product specification and features.

\section{Data Preparation}

In this step the unstructured format of the information is collected from the documentation and convert it to structured formats to propose recommendation to user more efficiently and effectively. The process is carried out by removing irrelevant data using text mining operations like removing stop words, markup tags etc. Stemming operation is also carried out in the document to have better accuracy.

\section{Similarity Finding}

In the similarity finding phase, the recommendation system gets the item information by taking in the items highlights from documentations. Highlights are watchwords that go about as brief item descriptors and enable items to be obviously recognized from each other.

A term, w, from a product's, $\mathrm{P}$, documentation is a component of $\mathrm{P}$ if the measure of data picked up by portraying $\mathrm{P}$ with $\mathrm{w}$ surpasses an edge esteem. The data increases are assessed by the Stochastic Complexity [22] measure, which demonstrates the measure of data contained in a grouping of information comparative with a model. It gauges the precision with which the information succession speaks to the model. The means of the TM-based Recommendation System to decide whether a word w from a documentation of sort $\mathrm{P}$ item is $\mathrm{P}$ 's component are represented underneath. The factors utilized in the significant conditions are as per the following:

$\mathrm{m}$ : The all out number of item documentations;

$\mathrm{m}+$ : The quantity of item documentations depicting type

$\mathrm{P}$ items;

mw: The quantity of item documentations containing the word w;

$\mathrm{m}+\mathrm{w}$ : The quantity of item documentations portraying type $\mathrm{P}$ items and containing the word w;

$\mathrm{m} \neg \mathrm{w}$ : The quantity of item documentations that don't contain the word $\mathrm{w}$;

$\mathrm{m}+\neg \mathrm{w}$ : The quantity of item documentations portraying type $\mathrm{P}$ items and not containing the word w;

the word w;

$$
\partial S C(w)=\left|\frac{1}{m}\left[S C\left(c^{m}\right)-\operatorname{SC}\left(c^{m} w\right)-S C\left(c^{m} \neg w\right)\right]\right|
$$

Finally, w is the key feature about the product that need to be recommended for the user.The features are formulated

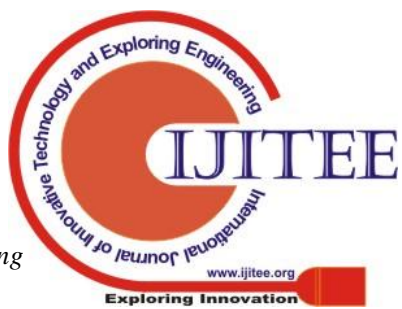


with rule-based classification method. IF <condition> THEN <action>, score $=\mathrm{x}$.

Thus after all the processing step this approach can perform its recommendation $f$ more effectively and efficiently.

\section{Generation of Recommendation}

Suggestions are created by activating principles whose conditions coordinate the terms in clients' data sources item inside the activity components of the laid-off guidelines are potential contender for proposal. For greater proposals, a running score is kept up for the up-and-comer item. The score of each activated principle is extra to the running score of the product appearing in its activity half, and items with the best scores are affixed to the counsel list .For instance, a client's information "Favored Phone Characteristics: in vogue has camera of dark shading" are reawakened to "style camera dark shading.

\section{Final Processing}

This stage enables to provide the recommendation system to refresh its information by a gradual learning approach .It figures the quantity of information picked up by the terms from their inclination particulars that didn't coordinate the states of any standards all through the counsel age. It verifies that terms is acclimated portray the product alternatives, and figures the relating decides that modify the development of the counsel quality.

\section{Discussion}

Table 1 uncovers that the proposed suggestion technique, in view of content mining, produces essentially preferable proposals over the conventional CF worldview. This improvement can be ascribed to its suggestion technique, which, in contrast to $\mathrm{CF}$, legitimately contrasts clients' determinations and item includes that has been educated. Besides, the proposed Recommendation System empowers clients to indicate their inclinations in literary organization, from which the precise learning of client inclinations is gotten, and better suggestions are produced to coordinate the inclinations. It additionally reduces the sparsity issue since it does not rely on customer profiles to generate suggestions, and along these lines, it can create precise proposals regardless of whether unequivocal client evaluations in their profiles are meager. The proposed content mining-based suggestion system is a lot quicker and more adaptable than the CF-based proposal technique.

The explanation is that the proposed suggestion approach is free of the quantity of client profiles since it doesn't make neighborhoods in the proposal system.

\section{CONCLUSION}

A book mining-based Recommendation System is displayed in this paper. It uncover that the proposed suggestion method reduces the versatility and sparsity confinements of conventional collective separating frameworks.. The proposed content mining-based Recommendation System doesn't make neighborhoods in its suggestion strategy, and thus, its suggestion philosophy is free of past client profiles.. Moreover, it doesn't experience the ill effects of the versatility issue which emerges in conventional shared separating based recommendation frameworks because of the high number of similitude computations that should be performed to find neighborhoods when the quantity of past client profiles increments.Since it catches the precise inclinations of the clients, including all subtleties and ambiguities, coordinating legitimately with the highlights of items that have been scholarly. Another advantage of the proposed content mining based suggestion procedure is that its proposals are not gotten from static client.

\section{REFERENCES}

1. Maizatul Akmar Ismail ,Damiasih Damiasih,Joko Sutopo,Tutut Herawan "A collaborative approach for research paper recommender system" Research article 2017

2. Robin van Meteren1 and Maarten van Someren "Using Content-Based Filtering for Recommendation1".

3. Jonathan L. Herlocker, Joseph A. Konstan, and John Riedl "Explaining Collaborative Filtering Recommendations".

4. Patrick Baudisch "Joining Collaborative and Contentbased Filtering".

5. R.J. Mooney, and L. Roy, "Content-based book recommending using learning for text categorization," In 5th ACM Conference on Digital Libraries, San Antonio, US, 2000, pp. 195-204.

6. A. Konstan, B.N. Miller, D. Maltz, J. L. Herlocker, L.R. Gordon, and J. Riedl, "GroupLens: applying collaborative filtering to Usenet news," Communications of the ACM, Vol. 40, pp. 77-87, 1997.

7. J.S. Breese, D. Heckerman, and C. Kadie, "Empirical analysis of predictive algorithms for collaborative filtering," In Proceedings of the Fourteenth Annual Conference on Uncertainty in Artificial Intelligence, pp. 43-52, July 1998.

8. L.H. Ungar, and D.P. Foster, "A formal statistical approach to collaborative filtering," In Proceedings of the Conference on Automated Learning and Discovery, 1998.

9. W. Lin, S.A. Alvarez, and C. Ruiz, "Collaborative recommendation via adaptive association rule mining," In Proceedings of WebKDD, 2000.

10. B. Sarwar, "Sparsity, scalability, and distribution in recommender systems," Ph.D. Thesis, University of Minnesota, 2001. 